\title{
Vibrations and Surface Generation in Slab Milling
}

\author{
Professor Dr.-Ing. U. Heisel - Sponsored by Professor Dr.-Ing. J. Milberg (1) \\ Received on January 15, 1994
}

\begin{abstract}
SUMMARY:
In slab milling the quality of the machined surface is directly influenced by the dynamic behaviour of the machine tool. In addition to the cycloidical microgrooves, the dynamic relative motion between the workpiece and tool is superimposed on the machined surface. In general these vibrations cannot be directly identified by investigating the machined surface. By the application of a special purpose milling cutter, microgrooves are generated which are identifiable and from which the relative motions in the cutting zone can be determined by surface topography measurement. It is shown how the groove structure is produced as a function of the kinematics of the process and the relative motions in the zone of cutting. An example based on experimental investigation is used to illustrate the procedure used to determine these relative motions based on analysis of the workpiece surface.
\end{abstract}

KEY WORDS: Machine Tools, Dynamic, Surface Generation

\section{Introduction}

On account of its high level of flexibility the milling process is one of the most commonly used chip removal manufacturing processes in industry. Based on the fundamental observations of the mechanisms of surface generation in slab milling as undertaken in the forties by Martellotti [5], Tlusty and Ismail [11] showed that for milling. which can be considered to be a non-stationary process (for process simulation purposes), a non-linear model must be selected. The two-dimensional dynamic model as shown in Figure 1 with $\mathrm{x}$ and $\mathrm{y}$ degrees of freedom permits the simulation of the superimposed vibrations of the cycloidical tooth motion of the milling cutter in the orthogonal plane. This helps in the observation of surface generation for both stable and unstable processes. Sato et al [7] published an FFT (Fast Fourier Transformation) analysis of surfaces affected by chatter. The phase lag of the chatter marks and the Fourier Coefficient could be identified. In further publications by Tlusty and Ismail [12] and Tlusty, Zaton and Ismail [14] in 1983 the general procedures for the calculation of machining forces for up-cut and down-cut milling were outlined. In this work the non-lineraties caused by entry and exit of the milling cutter to and from the workpiece as well as computer simulation of the stability of the milling process for uniformly and non-uniformly distributed teeth in the milling cutters is described. In [13] the spindle modes which occur in regard to the damping of the system through the process is presented and the corresponding observations in relation to the requirements for high speed milling are discussed.

Tsai and Sata [15] published the results of simulation of the milling process and milling cutter displacement for the case where several teeth of the cutter are in engagement and for conditions under which chatter takes place. Smith and Tlusty $[8,9,10]$ have reported on methods for the calculation and simulation of milling processes using further developed multi-degree of freedom models for consideration of the unstable, regenerative chatter effects. Minis et al [4] have reported on the determination of dynamic parameters by the measurement of structural vibrations and machining forces at the workpiece. The relative flexibility is determined by subtraction of two acceleration signals. In the work of Montgomery and Altintas [6] in 1991, a dynamic milling process is modelled having forces in two directions. Surface generation due to cycloidical motion with additional vibration superimposition is outlined. The influence of higher harmonic frequencies and vibrational effects on the machined surface are described. The most recent work from Ismail et al [2] in 1993 discusses the generation of milled surfaces taking into account run-out inaccuracies, milling cutter vibrations as well as tool wear. In addition to changing process parameters (cutting conditions, tool geometry etc.), one definite method of

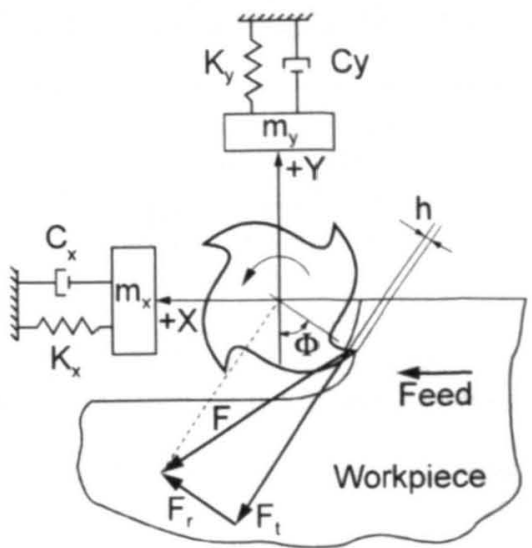

Fig. 1: Two-dimensional Dynamic Simulation Model by Ismail et. al. [2] respectively Smith and Tlusty [9]

influencing the process on the basis of the simulation results described above is through the optimisation of the dynamic behaviour of the machine tool. Suggestions for improvement could be through the reduction of exciting amplitudes by changing the directional orientation and possibly also the phase lag. Changes in the analysed vibrational forms by specific design improvements of specific components is particularly effective. For the determination of the transmission behaviour of the machine tool and the interference vibrations between tool and workpiece, an analysis of the elementary vibrational modes is presented. These, however, indicate the behaviour independent of the machining process. As against this, the operational vibration analysis shows those vibrations, which arise for the particular process and machine tool under specific machining conditions as super-imposed upon the modal vibrational signals $[3,16]$. Those vibrations which have a negative effect on the surface can only be experimentally determined if extensive effort is made using advanced measurement techniques.

\section{Surface Analysis and Vibrational Identification}

In a stable, static slab milling process the tooth engagement leaves its imprint as a part of a cycloid in the surface profile of the workpiece. A periodical microgroove structure is generated by sequential engagement of the cutter teeth. This structure is dependent on the number of teeth, the speed of revolution of the milling cutter and on the feed per tooth in the order of tooth engagement. It represents the primary parameter of surface roughness as defined in [17]. The tooth engagement time T compared with the time taken for one rotation of the cutter divided by the number of teeth is very small during effective surface generation with a microgroove width of $f_{2}$ (Figure 2). This means that 


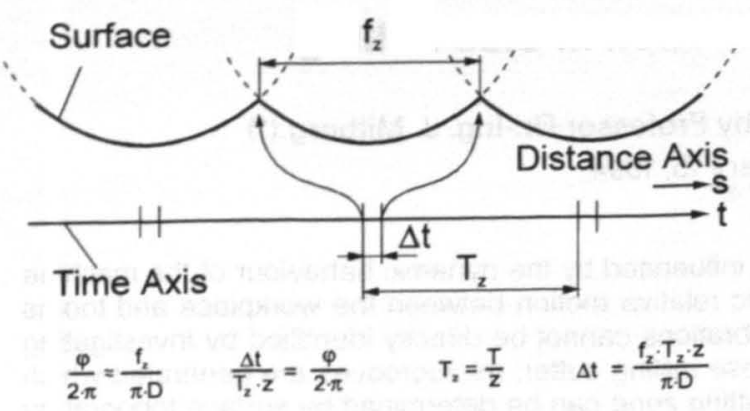

Fig. 2: The Relationship between the Time and Distance Axes in Surface Generation
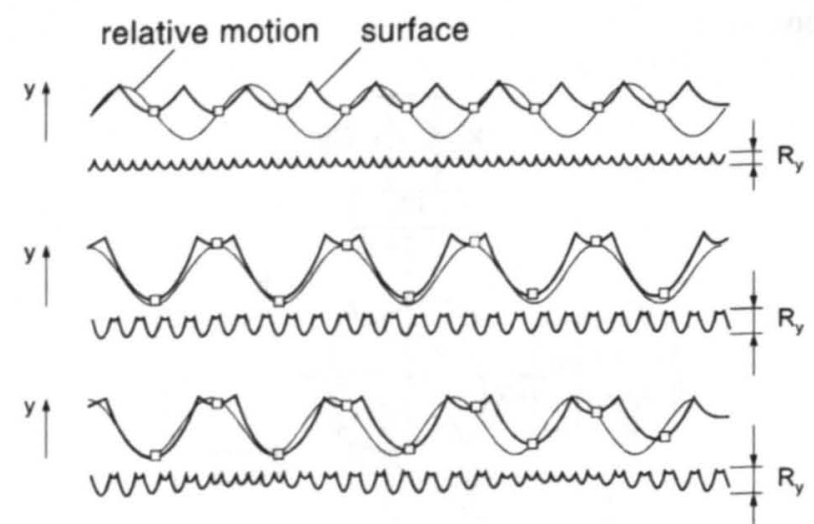

Fig. 3: The Effect of Phase Lag upon Generation of Waviness and Grooves

the respective relative motions can only be imprinted for very short and particularly discreet time intervals.

If a single tooth milling cutter $(z=1)$ is given consideration in regard to the surface analysis in a dynamic process, then different surface profiles are obtained depending upon amplitude, frequency and phase of the dynamic relative motion between workpiece and milling cutter. Figure 3 shows this for three examples of a constant, sinusoidal relative motion having different phase lags for a simulation model having the degree of freedom $y$. The strong influence of the dynamic components on the degree of roughness of the surface profile can be seen. Vibrations with the tooth engagement frequency as well as higher harmonic vibrations lead to a shifting of the overall position of the profile (Figure 4). The shape of the microgroove itself is only markedly influenced by much higher frequency signals. The different profile positions in a vertical direction
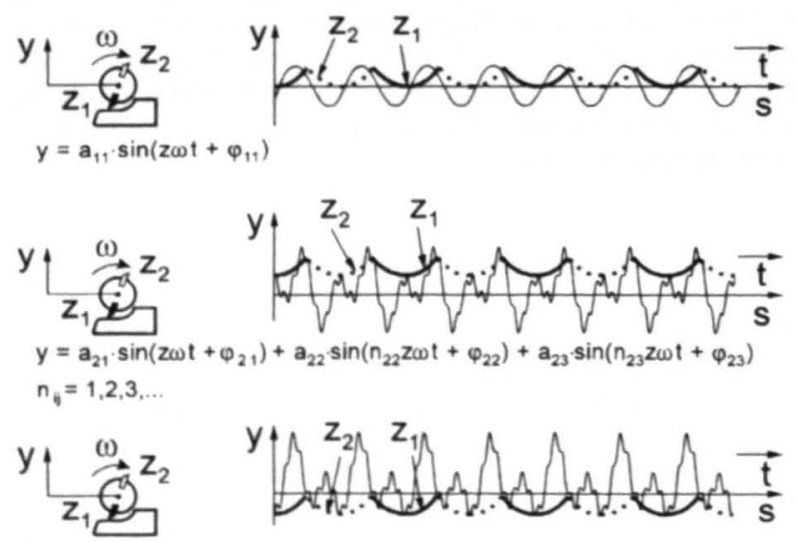

$y=a_{31} \cdot \sin \left(z \omega t+\varphi_{31}\right)+a_{32} \sin \left(n_{32} z \omega t+\varphi_{32}\right)+a_{33} \cdot \sin \left(n_{33} z \omega t+\varphi_{33}\right)$

$n_{i}=1,2,3$,

Fig. 4: Influence of different Harmonic Vibrations on Surface Generation in relation to the Tooth Engagement Frequency

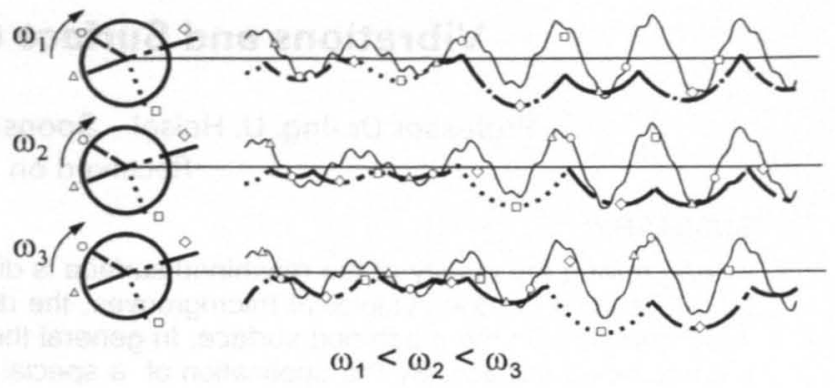

Fig. 5: A General Process with a four toothed, nonuniform divided Cutter with different tooth radiuses when Varying the Speed of Rotation

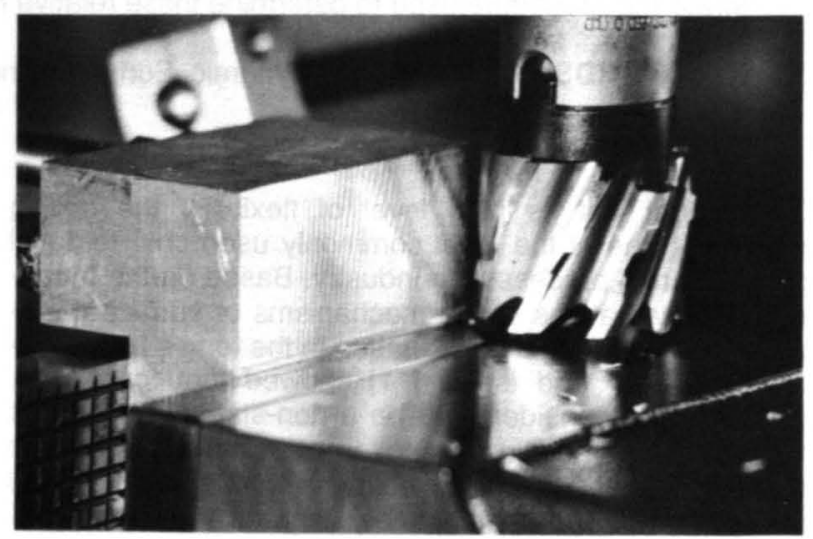

Fig. 6: Experimental Workpiece and Coded Cutting Tool with recessed Cutting Edges

normal to the workpiece surface shown in figure 4 , for instance, were generated by changing the phase position of individual harmonic components of the relative vibrations. If an actual standard milling process using a multi-tool cutter is observed (Figure 5), a different surface profile produced with various rotational speeds is found to occur for identical relative motions. This is because of the fact that the respective times for the important surface generation imprints of the tooth engagement occur at different times and thus, the microgrooves are generated on a different level corresponding to the current relative position. A similar situation can also be expected for changed relative motion. The actual vibrations which occur in stable and unstable processes are of a stochastic nature. Their imprint on the workpiece surface takes place at the respective discreet time intervals of tooth engagement. The extent and frequency of vibration for defined tooth engagement cannot be identified for a surface milled using a multi-tooth cutter.

In order to identify those vibrational characteristics which have an influence on the topography of a milled surface a

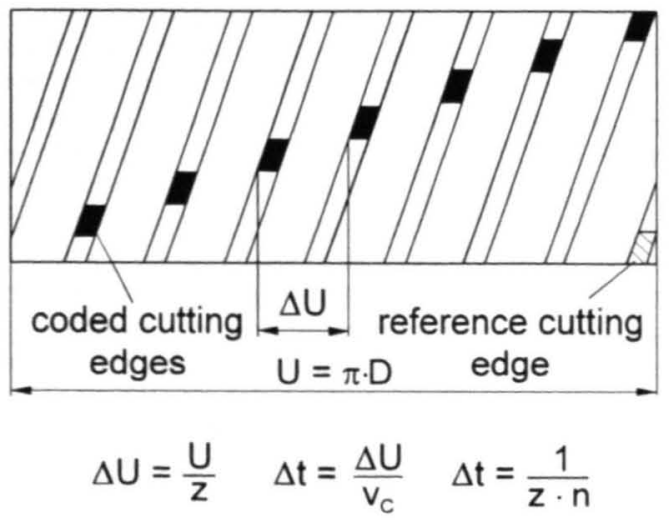

Fig. 7: The Circumferential Development of a Coded Cutting Tool 
specially prepared milling cutter was used in experiments which had specific cutting edges recessed respectively ground back (Figure 6). The edges are recessed only slightly such that the chip section and the resulting cutting forces are only negligibly affected. Figure 7 shows a development of such a tool prepared respectively coded in the way mentioned above with a number of single cutting edges which result in individually coded microgrooves. Each coded groove generates its own profile on the machined surface in a similar way to a single tooth cutter. The tooth engagement takes place in short succession and generates a number of microgrooves on the workpiece surface which correspond to the number of individual cutting edges (Figure 8).

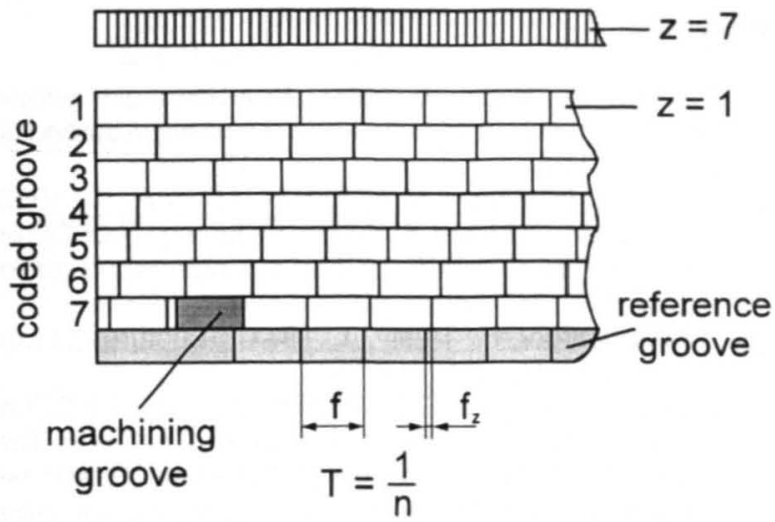

Fig. 8: The Imprint of the Coded Tool into the Workpiece Surface

The time interval between the engagement of individual teeth is defined for a uniform distribution of teeth about the milling cutter circumference and derives from the speed of rotation of the cutter and the number of individual teeth. In this way a discreet time interval is defined for each tooth engagement. In addition, it is clearly specified which particular tooth engagement can be associated with a particular element of the surface generated. By investigating successive tooth engagements it is possible to reconstruct the primary relative motions responsible for surface generation based on the surface profile of the individual microgrooves. The procedure for the identification of vibration by making use of the information associated with the profile of the machined surface for the simulated example of a milling cutter with seven cutting edges is shown in Figures 9 and 10. Making use of Fast Fourier Transformation (FFT), the relevant frequencies in regard to specific surface characteristics (in particular the low frequencies) can be obtained from the information generated.

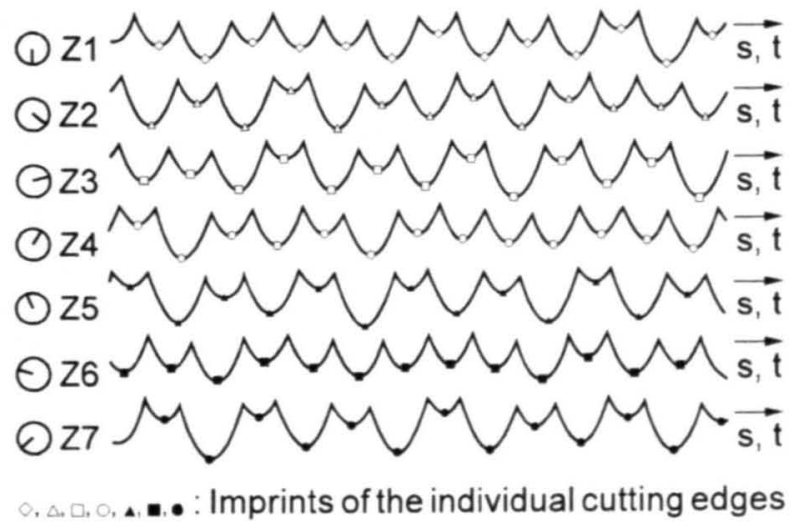

Fig. 9: Simulation of Surface Profiles of different Coded Microgrooves varying because of relative motions and im. print times

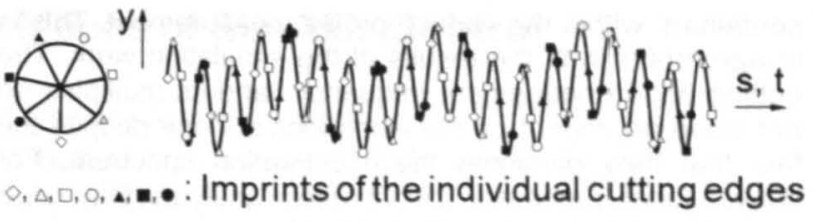

Fig. 10: Relative Motion between Workpiece and Cutter compiled out of seven Coded Microgrooves

\section{Experimental Verification}

In order to verify the procedure adopted above, a series of experiments was undertaken. In the following example a workpiece made of aluminium was used in conjunction with a milling cutter having seven cutting edges $(z=7)$ and one further reference edge. The milling cutter diameter was $D=40 \mathrm{~mm}$ and a speed of revolution $n=900$ RPM was used with a feed of $v_{f}=1.35 \mathrm{~m} / \mathrm{min}$. This feed was selected for technical reasons associated with the experiments. During machining, a vibration having a frequency of $22.5 \mathrm{~Hz}$ and an amplitude of 7 microns was externally applied using a vibration exciter. For comparison purposes, workpieces were prepared for which no vibration was transmitted from external sources. The surfaces generated for these workpieces did not exhibit features associated with the externally applied excitation.

Using a stylus type surface measurement instrument to determine the condition of the seven coded microgrooves it was possible to reconstruct the status of relative motion between the workpiece and cutting tool. The measurements of the reconstructed relative motion associated with differing time intervals can be related back to the individually coded cutting edges. Figure 11 shows the calculated and measured surface profile for a single coded

a)

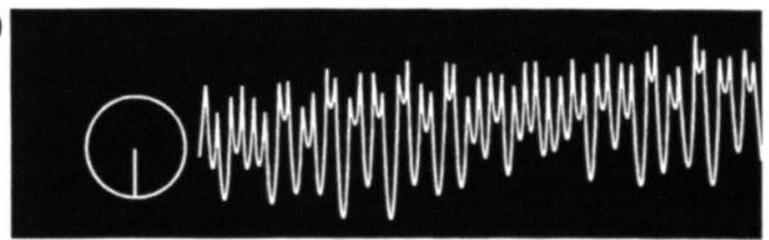

b)

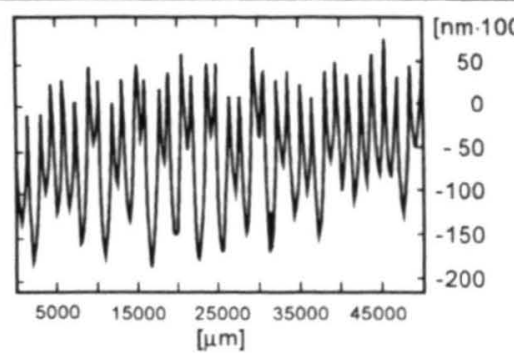

Fig. 11: Calculated (a) and Measured (b) Surface Profile shown for a single Coded Microgroove

microgroove. Both profiles can be seen to have very similar characteristics.

The frequency analysis undertaken for this relative motion which occurred at equi-distant time intervals results in a vibrational frequency of $22.8 \mathrm{~Hz}$ with an amplitude of 7 microns. A second frequency of $4.35 \mathrm{~Hz}$ with an amplitude of 3 microns also occurs. The latter frequency which was not transmitted from external sources can be associated with the feed motion and could also be identified for those machining experiments which were not subjected to external excitement. Such low frequencies would not be identifiable when using accelerometers and become lost in the system noise.

The measured rotational frequency of the cutter of $15 \mathrm{~Hz}$ as shown by the displacement spectrum was not 
contained within the surface profile measurement. This is in agreement with the results of the simulation work. The cutting edge engagement frequency and its multiples do not leave an imprint on the workpiece surface despite the fact that they dominate the acceleration spectrum. For conventional processes of vibration analysis this often leads to incorrect optimisation objectives.

The achievable accuracy in the process described above in which the relative motions in the zone of cutting are estimated by analysis of the surface generated is dependent on the number of tooth engagements which take place during one rotation of the milling cutter and on the imprint which represents the primary parameter within the surface profile.

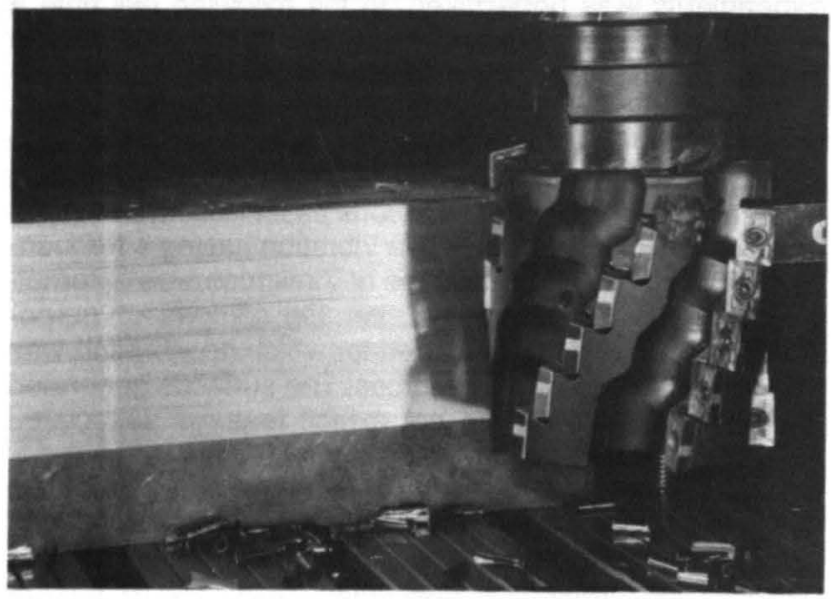

Fig. 12: A Tungsten Carbide Indexible Insert Milling Cutter with Recessed Cutting Edges. (Machining Conditions: Workpiece Material Ck 45, D=80 mm, $n=600 \mathrm{~min}^{-1}$, $f_{z}=0,3 \mathrm{~mm}, a_{\theta}=5 \mathrm{~mm}, v_{c}=150 \mathrm{mmin}^{-1}, a_{p}=70 \mathrm{~mm}$ )

For the experiments described above the number of imprinted tooth engagements corresponds to the number of teeth in the milling cutter which was $z=7$. The sampling frequency achievable in the analysis corresponds to the number of teeth and the rotational frequency of the milling cutter i.e. $\mathbf{z} \cdot \mathbf{w}$. As stated in Shannon's sampling theorem, the formula $f_{M \max } \leq \frac{z}{2} \cdot \frac{\omega}{2 \cdot \pi}$ applies for the maximum measurable sampling frequency. For the machining parameters outlined above this yields a frequency of $52.5 \mathrm{~Hz}$.

This means that the higher frequency components of the relative motion do not leave their imprint in a reconstructable manner on the surface profile. In order to increase the accuracy of analysis the number of teeth available or the rotational frequency has to be increased. This point is of particular interest in relation to experiments under high speed machining conditions.

The milling cutter shown in Figure 12 having tungsten carbide indexible inserts includes $5 \cdot 5=25$ teeth. For the machining conditions outlined in the figure title a maximum measurable frequency of $f_{M \max }=125 \mathrm{~Hz}$ is obtained. A prerequesite for this is that 25 individually identifible coded microgrooves were generated each having a slightly recessed cutting edge on the individual indexible inserts. On account of the milling cutter design and in particular due to the arrangement of the indexible inserts the relative motions generated during machining with this milling cutter do not result in equidistant time intervals. This has to be taken into account when evaluating the frequency and amplitude using FFT and should be corrected in a suitable manner in advance.

In conclusion it can be stated that the identification of vibration as determined by the above method from the surface profile of a workpiece represents very accurately the true relative motion between workpiece and cutting tool which takes place during surface generation.

\section{Conclusions}

The method of surface analysis described in this paper permits the identification of the dynamic relative motion between the workpiece and milling cutter in slab milling which takes place during surface generation. The critical frequencies in relation to influence on surface generation and surface roughness can be determined. In this way a defined procedure is made available for a direct analysis of the mechanisms of surface generation in specific applications. This provides a basis for machine tool optimisation [1]. The above results have already been confirmed. One further objective could be to extend these results to models for other machining processes.

\section{References}

[1] Fischer, A., Walz, J., 1992, Bearbeitungsergebnis zur dynamischen Maschinenoptimierung nützen, dima, 46:20-25.

[2] Ismail, F., Elbestawi, M.A., Du, R., Urbasik, K., 1993 Generation of Milled Surfaces Including Tool Dynamics and Wear, ASME Journal of Engineering for Industry, 115: 245-252.

[3] Koenigsberger, F., Tlusty, J., 1971, Structures of Machine Tools, Pergamon Press.

[4] Minis, I.E., Magrab, E.B., Pandelidis, I.O., 1990, Improved Methods for the Prediction of Chatter in Turning, Part 1: Determination of Structural Response Parameters, ASME Journal of Engineering for Industry, 112: 12-20.

[5] Martellotti, M.E., 1941, An Analysis of the Milling Process, Transactions of the ASME, 63: 667-700.

[6] Montgomery, D., Altintas, Y., 1991, Mechanism of Cutting Force and Surface Generation in Dynamic Milling, ASME Journal of Engineering for Industry, 113: $160-168$.

[7] Sato, H., O-hori, M., Tsuwa, H., 1981, Characteristics of Two-dimensional Surface Roughness-Taking Selfexcited Chatter Marks as Objective, CIRP Annals, 30/1: 481-486.

[8] Smith, S., Tlusty, J., 1993, Efficient Simulation Programs for Chatter in Milling, CIRP Annals, 42/1: 463-466.

[9] Smith, S., Tlusty, J., 1991, An Overview of Modeling and Simulation of the Milling Process, ASME Journal of Engineering for Industry, 113: 169-175.

[10] Smith, S., Tlusty, J., 1990, Update on High-Speed Milling Dynamics, ASME Journal of Engineering for Industry, 112: 142-149.

[11] Tlusty, J., Ismail, F., 1981, Basic Non-Linearity in Machining Chatter, CIRP Annals, 30/1: 299-304.

[12] Tlusty, J., Ismail, F., 1983, Special Aspects of Chatter in Milling, ASME Journal of Vibration, Acoustics, Stress, and Reliability in Design, 105: 24-32.

[13] Tlusty, J., 1986, Dynamics of High-Speed Milling, ASME Journal of Engineering for Industry, 108: 59-67.

[14] Tlusty, J., Zaton, W., Ismail, F., 1983, Stability Lobes in Milling, CIRP Annals, 32/1: 309-313.

[15] Tsai, M.D., Takata, S., Inui, M., Kimura, F., Sata T., 1990 , Prediction of Chatter Vibration by Means of a Model-Based Cutting Simulation System, CIRP Annals, 39/1: 447-450.

[16] Weck, M., Teipel, K., 1977, Dynamisches Verhalten spanender Werkzeugmaschinen, Springer-Verlag, Berlin Heidelberg New York.

[17] ISO 4287/1: 1984, Surface roughness - Terminology, Part 1: Surface and its parameters. 